\title{
Heuristics for the economic lot scheduling problem with returns ${ }^{\text {is }}$
}

\author{
Ruud Teunter $^{\mathrm{a}}$, Ou Tang ${ }^{\mathrm{b}, *}$, Konstantinos Kaparis ${ }^{\mathrm{a}}$ \\ a Department of Management Science, Lancaster University Management School, Lancaster LA1 4YX, UK \\ ${ }^{\mathrm{b}}$ Department of Management and Engineering, Linköping University, SE-581 83 Linköping, Sweden
}

\section{A R T I C L E I N F O}

Available online 23 August 2008

\section{Keywords:}

ELSP

Returns

Remanufacturing

Reverse logistics

\begin{abstract}
A B S T R A C T
We study the multi-item economic lot scheduling problem (ELSP) with two sources of production: manufacturing of new items and remanufacturing of returned items. Manufacturing and remanufacturing operations are performed on the same production line. Tang and Teunter [2006. Economic lot scheduling problem with returns. Production and Operations Management 15 (4), 488-497.] recently presented a complex algorithm for this problem that determines the optimal solution within the class of policies with a common cycle time and a single (re)manufacturing lot for each item in each cycle. This algorithm is rather complex and time consuming, combining a large MIP formulation with a search procedure, and may therefore not always be practical. In this paper, we deal with this type of problems and propose simple heuristics that are very fast and can be applied in a spreadsheet package. A large numerical study shows that the heuristics provide close to optimal solutions.
\end{abstract}

(c) 2008 Elsevier B.V. All rights reserved.

\section{Introduction}

Closed-loop supply chain management has emerged as a new research area due to strict legislations, potential profit margins of reusing return products and customers' awareness of environment-friendly products. To cope with the development of this new business environment, some conventional models need to be reinvestigated to support decision making. This is particularly important at the operational level (Guide, 2000).

Motivated by a real-life study of a company that (re)manufactures car parts for the service market, Tang and Teunter (2006) recently extended the well-known economic lot scheduling problem (ELSP, Bomberger, 1966; Elmaghraby, 1978) to include the return flows. They presented an algorithm that determines the optimal solution within the class of policies with a common cycle

\footnotetext{
Paper submitted for presentation at the 14th International Symposium on Inventories, Budapest, Hungary, 21-25 August 2006.

* Corresponding author. Tel.: +4613 281773; fax: +4613281101.

E-mail address: ou.tang@liu.se (O. Tang).
}

time and a single (re)manufacturing lot for each item in each cycle. Their algorithm combines a search for the optimal cycle time with a mixed integer programming (MIP) formulation given a fixed cycle time. A practical drawback of the algorithm is that the MIP is rather complex and its programming therefore tedious. Firms may not have the programming knowledge or software (e.g. CPLEX) to implement the algorithm. Even if they do, applying the algorithm may be too time consuming depending on the problem size (number of products). In this respect, it is important to bear in mind that hybrid manufacturing/remanufacturing processes face many uncertainties, especially fluctuating demand and return rates, and hence there is a need for frequently updating model parameters and recalculating solutions.

In this paper, we therefore propose and examine heuristics for the economic lot scheduling problem with returns (ELSPR). We present heuristic approaches, which can be readily implemented in practice. The performances of these heuristics are tested in a large numerical investigation.

Literature mostly relevant to the ELSPR can be divided into two categories. First there exist a body of literature 


\section{Nomenclature}

$N \quad$ number of products

$D_{i} \quad$ constant demand rate for item $i=1,2, \ldots, N$, units/time unit

$h_{i}^{\mathrm{s}}\left(h_{i}^{\mathrm{r}}\right) \quad$ inventory holding cost for serviceable (recoverable) inventory, \$/unit/time unit

$K_{i}^{\mathrm{m}}\left(K_{i}^{\mathrm{r}}\right)$ setup costs for manufacturing (remanufacturing), \$/lot

$P_{i}^{\mathrm{m}}\left(P_{i}^{\mathrm{r}}\right)$ manufacturing (remanufacturing) rate, units/ time unit $s_{i}^{\mathrm{m}}\left(s_{i}^{\mathrm{r}}\right) \quad$ setup time of manufacturing (remanufacturing), time units

$T \quad$ common cycle time, time units

$x_{i}^{\mathrm{m}}\left(x_{i}^{\mathrm{r}}\right) \quad$ time at which the manufacturing (remanufacturing) of item $i$ starts, time units

$\beta_{i} \quad$ constant return proportion of item $i, 0 \leqslant \beta_{i}<1$

$f(z)$

$[z]^{+} \quad=\max \{z, 0\}$

$[z]^{-} \quad=\max \{-z, 0\}$ dealing with conventional ELSP problem, with discussion of model formulation and solution algorithms (Bomberger, 1966; Elmaghraby, 1978; Hsu, 1983; Davis, 1990, among many others). Secondly, there are emerging studies of lot sizing problem in remanufacturing of returns with focus on the interaction of manufacturing and remanufacturing decisions (Richter, 1996; Teunter, 2001; Koh et al., 2002; Teunter, 2004). These studies are all limited to single item cases though. For a more comprehensive literature review in the above two areas, and for a detailed description of a real-life ELSPR case, we refer to Tang and Teunter (2006).

The remainder of this paper is structured as follows. In Section 2, we describe the ELSPR production system. In Section 3, we review those results from Tang and Teunter (2006) that provide some of the basis for the heuristics. In Section 4, solution principles and heuristics are presented. A numerical study is carried out in Section 5. Finally, in Section 6, we draw our study conclusion and make suggestions for future studies.

\section{Description of the production system}

We study a production system where several products (models) are produced by both manufacturing and remanufacturing. Products from manufacturing and remanufacturing have no quality difference and they are pooled into the same serviceable stock. Returns are collected into recoverable stock and consumed when remanufacturing starts. Manufacturing and remanufacturing compete for the same resource, for instance machine capacity. As it is common in the ELSP literature, we assume constant demand, return, manufacturing and remanufacturing rates, as well as sequence-independent setup costs/times.

As Tang and Teunter (2006), we restrict our attention to the common cycle time policies, i.e. policies with the same cycle time for all products and with a single manufacturing lot and a single remanufacturing lot for each item in each cycle. The objective is to find the optimal policy: that is, to find the cycle time and the production start times for (re)manufacturing lots that minimize the total cost per time unit, which include the setup costs for manufacturing and remanufacturing, the holding cost (per item per time unit) for returned products, and the holding cost for serviceable products.

\section{Review of relevant results from Tang and Teunter (2006)}

In this section, we review some of the results of Tang and Teunter (2006) that will provide the basis for the heuristics presented in Section 4.

A key difference in the (common cycle time) analysis of the ELSPR as opposed to the traditional ELSP is that the sequencing of lots matters. For the ELSP, any schedule/ sequence leads to the same stock level patterns and average stock levels. For the ELSPR, however, the scheduling does matter, because the relative timing of manufacturing and remanufacturing lots for a product affects its stock patterns and levels. For similar-sized lots of the two types, for instance, it is obviously better to schedule them so that one does not start shortly after the other. The relevance of scheduling for the ELSPR makes it a more complex problem than the ELSP. An additional difficulty for the ELSPR is that recoverable inventory is considered as well.

The first result of Tang and Teunter (2006) extends the minimum cycle time restriction for the traditional ELSP to give

$T \geqslant T_{\min }=\frac{\sum_{i=1}^{N}\left(s_{i}^{\mathrm{m}}+s_{i}^{\mathrm{r}}\right)}{1-\sum_{i=1}^{N} D_{i}\left(\left(1-\beta_{i}\right) / P_{i}^{\mathrm{m}}+\beta_{i} / P_{i}^{\mathrm{r}}\right)}$,

which indicates that the common cycle time should be long enough to cover the total production time and setup time for both manufacturing and remanufacturing. When return rate $\beta_{i}$ is reduced to zero and the remanufacturing setups $s_{i}^{r}$ are removed, the above expression reduces to the cycle time constraint for the traditional ELSP.

The second result splits the total average cost per time unit TC into the so-called ideal cost (IC) and additional cost $(\mathrm{AC})$, i.e.

$\mathrm{TC}=\mathrm{IC}+\mathrm{AC}$.

The ideal cost includes setup costs, serviceable and recoverable holding costs when the time gap between manufacturing and remanufacturing lots are ideal, i.e. when the serviceable inventory always drops to zero if a production lot of either type starts. This happens when the time between the start of a remanufacturing lot and the successive manufacturing lot is $T\left(1-\beta_{i}\right)$ and hence the time between the start of a manufacturing lot and the successive remanufacturing lot is $T \beta_{i}$. In this case, the total 
demands during these intervals, $D_{i} T\left(1-\beta_{i}\right)$ and $D_{i} T \beta_{I}$, respectively, are exactly equal to the remanufacturing and manufacturing lot sizes. The ideal cost can be written as

$$
\begin{aligned}
\mathrm{IC}= & \sum_{i=1}^{N} \frac{K_{i}^{\mathrm{m}}+K_{i}^{\mathrm{r}}}{T}+h_{i}^{\mathrm{r}} \frac{\mathrm{TD}_{i} \beta_{i}}{2}\left(1-\frac{D_{i} \beta_{i}}{P_{i}^{\mathrm{r}}}\right) \\
& +h_{i}^{\mathrm{s}} \frac{\mathrm{TD}_{i}}{2}\left(\beta_{i}^{2} \frac{P_{i}^{\mathrm{r}}-D_{i}}{P_{i}^{\mathrm{r}}}+\left(1-\beta_{i}\right)^{2} \frac{P_{i}^{\mathrm{m}}-D_{i}}{P_{i}^{\mathrm{m}}}\right) .
\end{aligned}
$$

Thus, IC is also the lower bound of the total cost. From Eq. (3), we can also derive the lower bound of the problem total cost IC $^{*}$ with the cycle time $T$ equals
With the cycle time fixed, what remains is to determine the production schedule, i.e. the timing of the production lots in a cycle. Next, we will introduce three more principles for doing so.

Principle 2 generates an initial schedule that starts a cycle with all manufacturing lots, followed by all remanufacturing lots. Recall from Section 3 that $T\left(1-\beta_{i}\right)$ is the ideal time between manufacturing and remanufacturing of item $i$, which is obviously decreasing in $\beta_{i}$. Principle 2 therefore schedules the manufacturing lots in ascending order of $\beta_{i}$, creating the largest time intervals where needed until remanufacturing starts. Once all manufacturing lots are scheduled, the (conditional on this

$$
T_{\mathrm{IC}}=\sqrt{\frac{2 \sum_{i=1}^{N}\left(K_{i}^{\mathrm{m}}+K_{i}^{\mathrm{r}}\right)}{\sum_{i=1}^{N} D_{i}\left(h_{i}^{\mathrm{r}} \beta_{i}\left(1-D_{i} \beta_{i} / P_{i}^{\mathrm{r}}\right)+h_{i}^{\mathrm{s}}\left(\beta_{i}^{2}\left(1-D_{i} / P_{i}^{\mathrm{r}}\right)+\left(1-\beta_{i}\right)^{2}\left(1-D_{i} / P_{i}^{\mathrm{m}}\right)\right)\right)}} .
$$

Of course due to the capacity and scheduling constraints, ideal timing of all manufacturing and remanufacturing lots for all products is not always feasible. This leads to a positive serviceable inventory level at the time production lot starts, and consequently an extra cost

$$
\begin{aligned}
\mathrm{AC}= & \sum_{i=1}^{N} h_{i}^{\mathrm{s}} D_{i}\left(\left(1-\beta_{i}\right)\left[f\left(x_{i}^{\mathrm{r}}-x_{i}^{\mathrm{m}}\right)-T\left(1-\beta_{i}\right)\right]^{+}\right. \\
& \left.+\beta_{i}\left[f\left(x_{i}^{\mathrm{r}}-x_{i}^{\mathrm{m}}\right)-T\left(1-\beta_{i}\right)\right]^{-}\right) .
\end{aligned}
$$

Here, $f\left(x_{i}^{\mathrm{r}}-x_{i}^{\mathrm{m}}\right)$ is the time between the starts of successive manufacturing and remanufacturing lots of the same product, and $T\left(1-\beta_{i}\right)$ is the ideal time, so that $f\left(x_{i}^{\mathrm{r}}-x_{i}^{\mathrm{m}}\right)-T\left(1-\beta_{i}\right)$ is the deviation from the ideal time. It is intuitively obvious that a larger deviation leads to a larger increase in AC, and (5) shows that the increase is in fact linear in the size of the deviation.

The numerical results in Tang and Teunter (2006) indicate that the optimal cycle time is close to the ideal cycle time if that is feasible and close to the minimum cycle time otherwise. Hence, the optimal cycle time seems to be almost independent of the sequence in which lots are produced. We will use this insight in Section 4 for developing heuristics.

\section{Heuristics}

A solution of the ELSPR is characterized by the cycle time (and the associated lot sizes) and the schedule. Recall from Section 3 that the optimal cycle seems to be almost independent of the schedule. The heuristics that we will propose therefore all use a two-step approach that first fixes the cycle time and then determines all production/ remanufacturing starting times. Recall further that the optimal cycle time is close to the ideal cycle time if that is feasible and close to the minimum cycle time otherwise, which leads to the following heuristic principle for setting the cycle time.

Principle 1-Cycle time. Set the cycle time equal to $\max \left\{T_{\min }, T_{\mathrm{IC}}\right\}$. manufacturing schedule) ideal starting times $x_{i}^{\mathrm{m}}-s_{i}^{\mathrm{r}}+$ $T\left(1-\beta_{i}\right)$ for all remanufacturing lots are known as well. The ideal sequencing of remanufacturing lots is therefore in descending order of $x_{i}^{\mathrm{m}}-s_{i}^{\mathrm{r}}+T\left(1-\beta_{i}\right)$.

Principle 2-Initial production sequence. Schedule all manufactures lots first in ascending order of the return rate $\beta_{i}$, starting with the first setup at time 0 and without slack time in between the lots. Then schedule all remanufacture lots in descending order of $x_{i}^{\mathrm{m}}-s_{i}^{\mathrm{r}}+T\left(1-\beta_{i}\right)$.

Fig. 1 illustrates the initial solution graphically for the case where $\beta_{3}<\beta_{2}<\beta_{1}<\beta_{4}$.

After the initial schedule is determined, we have two principles for improving it: either by swapping 'neighbouring' lots (Principle 3 ) or by redistributing the slack time that is initially concentrated at the end of the cycle (Principle 4). Both principles aim at reducing the deviation from the ideal time between the starts of manufacturing and remanufacturing lots $f\left(x_{i}^{\mathrm{r}}-x_{i}^{\mathrm{m}}\right)-T\left(1-\beta_{i}\right)$. We will discuss them in this order.

Obviously, swapping neighbouring lots to improve the timing for a specific item may worsen the timing for the other item involved in the swap (assuming that the two lots are not of the same product). Hence, rather than randomly selecting lots to swap, we prefer to improve the schedule for those products for which timing has a large effect on the total cost. The additional cost expression (5) shows that this effect is proportional to $h_{i}^{\mathrm{s}} D_{i}$, and this leads to the following swapping principle.

Principle 3 - Swapping neighbouring lots. Find the item with the highest value of $h_{i}^{\mathrm{s}} D_{i}$. Determine the effects on $A C$ of swapping either the manufacturing or remanufacturing lot with one of its neighbours (giving 4 possible swaps). If AC can be reduced, then perform the swap that gives the largest cost reduction and repeat the procedure for this item. Otherwise,

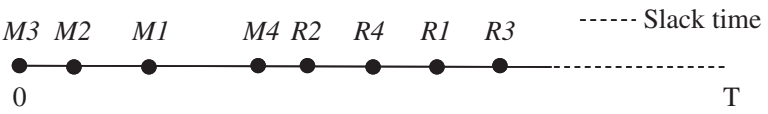

Fig. 1. An example of the initial schedule. 


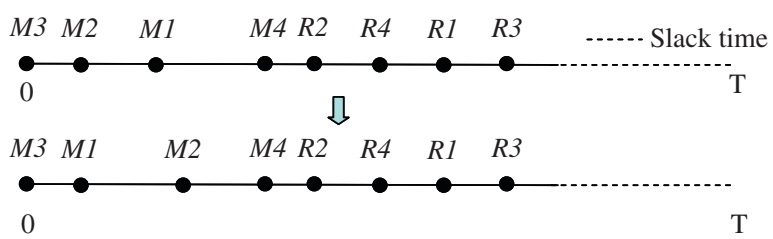

Fig. 2. Swapping the production sequence.

continue with the item with next highest value of $h_{i}^{\mathrm{s}} D_{i}$. Stop when all items have been examined.

Fig. 2 provides an example of swapping. Assuming item 1 has the highest value of $h_{i}^{\mathrm{s}} D_{i}$, we switch the order sequence M1-M2 to see the possibility of reducing AC. Other alternatives (not shown in the figure) are switching M1-M4, R1-R4 and R1-R3.

Next, we discuss the second improvement principle based on redistributing slack time. Products are considered in the order of time at which the second lot (manufacturing or remanufacturing) starts. The setup of the considered lot is postponed by inserting slack time before it, but only if doing so reduces (AC), which can easily be checked. The lot is shifted to its ideal starting point if there is sufficient slack time left (at the end of the cycle) and otherwise by the available amount of slack time.

Principle 4 -Using slack time. Locate the start times of second production lot (either manufacturing or remanufacturing) for all products. These times are recursively adjusted one by one in the order in which the lots are sequenced, i.e. starting with the item for which the second lot starts earliest. If the ideal time between the manufacturing and remanufacturing lots of this product is smaller than the actual one, insert no slack time. Otherwise, delay the start of second lot until the ideal start time or until no more slack time is available (whichever happens first), but only if that leads to a cost reduction. Update the remaining slack time accordingly. The approach stops when all products have been examined or slack time is reduced to zero.

Note that the initial slack time determined is positive if $T_{\mathrm{IC}}>T_{\min }$ and zero if $T_{\mathrm{IC}} \leqslant T_{\min }$.

Fig. 3 illustrates the idea. Item 2 is the first item for which the second lot (remanufacturing in this case) starts. We insert the slack time before $\mathrm{R} 2$ to see if $\mathrm{AC}$ can be improved. We have to be aware that the start time of all lots after R2 will shift the same amount. However, when we move to the next pair and insert slack time again (for instance before R4 in the next step), the start time of the checked items will not change (the distance between M2 and $\mathrm{R} 2$ is constant). This leads to a quick convergence of the algorithm.

Based on the above principles, we propose four heuristics:

- Heuristic A: Principles $1+2$

- Heuristic B: Principles $1+2+3$

- Heuristic C: Principles $1+2+4$

- Heuristic D: Principles $1+2+3+4$

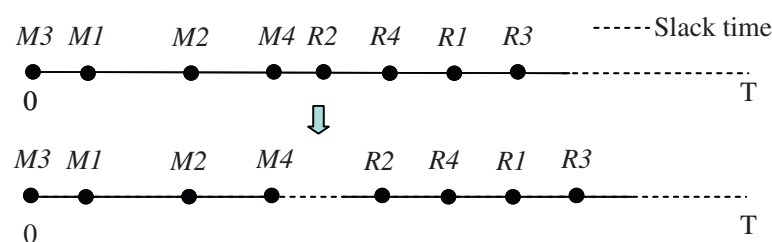

Fig. 3. Inserting slack time.

Obviously, Heuristic A is the simplest and D the most complex, but all can be implemented in a spreadsheet. Heuristic A is also simple enough to be done by hand. Note that we do not consider the option of applying the slack time principle first, followed by the swapping principle. This would be more complex than the proposed Heuristic D. Time slacks between lots create more alternatives for swapping, i.e. the swapped start times can be in the range of slack time and a thorough search is needed to obtain the best solution. This complicates the heuristic and, according to our experiments, does not considerably improve the results. In Section 5, we will compare the performances of the heuristics in an extensive numerical study.

\section{Numerical study}

\subsection{Generation of examples}

In a previous study (Tang and Teunter, 2006), industrial data were collected for a water pump (re)manufacturing system. However, that data set shows a capacity utilization around $80 \%$, which prevents us from examining the performance of each heuristic with respect to capacity utilization. Furthermore, return rates are 20\% for all pumps and other relevant parameters the same for all items as well. Therefore, we choose not to use these restricted data for testing the heuristics.

Instead, we generate examples by extending the famous Bomberger (1966) lot sizing problem with manufacturing only, which is often used as a benchmark for testing the quality of policy restrictions (e.g. a common cycle time) and of heuristic solution approaches.

Thus, the data for base example 0 are generated as follows from the original Bomberger problem. For each item:

- the demand rates are the same as in the original problem,

- the manufacturing and remanufacturing setup times are both equal to the original setup time,

- the manufacturing and remanufacturing setup costs are equal to the original setup cost multiplied with a random number between 0 and 2 ,

- the manufacturing and remanufacturing production rates are equal to the original production rate multiplied with a random number between 0 and 2 ,

- the serviceables holding cost is the original holding cost,

- the returns holding cost is the original holding cost multiplied with a random number between 0 and 1 
(The returns holding cost should be smaller than the serviceable holding cost, since remanufacturing adds value to an item. Interested readers are referred to Teunter et al. (2000) for a general discussion on how to set holding cost rates in models with remanufacturing.),

- the return rate is a random number between 0 and 1 .

For illustration, Table 1 shows the rounded parameter settings for Example 1.
The complete set of examples is available from the authors. We remark that we did not simply pick the first 120 examples that were generated. First, generated examples that did not satisfy the assumption that production rates are larger than demand rates were discarded. Second, to get a good spread in terms of capacity utilization (excluding setup times), we discarded examples so that we ended up with

- Examples 1-30: capacity utilization is below 50\%;

Table 1

Parameters settings for example 1

\begin{tabular}{|c|c|c|c|c|c|c|c|c|c|c|}
\hline \multirow{2}{*}{$\begin{array}{l}\text { Item no. } \\
i\end{array}$} & \multicolumn{3}{|c|}{ Manufacturing } & \multicolumn{3}{|c|}{ Remanufacturing } & \multicolumn{2}{|c|}{ Holding cost $\left(\times 10^{7}\right)$} & \multirow{2}{*}{$\begin{array}{l}\text { Demand } \\
\text { rate } \\
D_{i}\end{array}$} & \multirow{2}{*}{$\begin{array}{l}\text { Return } \\
\text { ratio } \\
\beta_{i}\end{array}$} \\
\hline & $\begin{array}{l}\text { Setup } \\
\text { cost, } K_{i}^{\mathrm{m}}\end{array}$ & $\begin{array}{l}\text { Setup } \\
\text { time, } s_{i}^{\mathrm{m}}\end{array}$ & $\begin{array}{l}\text { Manu. } \\
\text { rate, } P_{i}^{\mathrm{m}}\end{array}$ & $\begin{array}{l}\text { Setup } \\
\text { cost, } K_{i}^{\mathrm{r}}\end{array}$ & $\begin{array}{l}\text { Setup } \\
\text { time, } s_{i}^{\mathrm{r}}\end{array}$ & $\begin{array}{l}\text { Reman. } \\
\text { rate, } P_{i}^{\mathrm{r}}\end{array}$ & $\begin{array}{l}\text { Serv. } \\
\text { Inven., } h_{i}^{\mathrm{s}}\end{array}$ & $\begin{array}{l}\text { Recov. } \\
\text { Inven., } h_{i}^{\mathrm{r}}\end{array}$ & & \\
\hline 1 & 2 & 0.192 & 15,715 & 12 & 0.031 & 54,700 & 13 & 17 & 67 & 0.44 \\
\hline 2 & 9 & 0.148 & 5471 & 37 & 0.224 & 3308 & 774 & 111 & 10 & 0.71 \\
\hline 3 & 3 & 0.473 & 7767 & 2 & 0.357 & 8689 & 359 & 26 & 108 & 0.95 \\
\hline 4 & 12 & 0.053 & 9820 & 5 & 0.02 & 25,743 & 307 & 295 & 226 & 0.86 \\
\hline 5 & 60 & 0.746 & 697 & 132 & 0.16 & 523 & 16,353 & 195 & 19 & 0.73 \\
\hline 6 & 37 & 0.426 & 20,180 & 153 & 0.167 & 3259 & 774 & 679 & 12 & 0.65 \\
\hline 7 & 47 & 1.286 & 2988 & 239 & 0.204 & 908 & 2875 & 1900 & 10 & 0.39 \\
\hline 8 & 218 & 0.447 & 1263 & 107 & 0.406 & 388 & 39,034 & 7089 & 6 & 0.72 \\
\hline 9 & 45 & 0.954 & 1129 & 150 & 0.906 & 525 & 3983 & 905 & 15 & 0.36 \\
\hline 10 & 11 & 0.016 & 7737 & 7 & 0.167 & 6811 & 303 & 52 & 134 & 0.64 \\
\hline
\end{tabular}

Table 2

Output for examples $1-30$ with capacity utilization $\mathrm{CU}<50 \%$

\begin{tabular}{|c|c|c|c|c|c|c|c|c|c|c|}
\hline \multirow[t]{2}{*}{$i$} & \multirow[t]{2}{*}{$T_{\min }$} & \multirow[t]{2}{*}{$T_{I C}$} & \multirow[t]{2}{*}{$T^{*}$} & \multirow[t]{2}{*}{ CU (\%) } & \multirow[t]{2}{*}{ IC } & \multirow[t]{2}{*}{$\mathrm{TC}^{*}$} & \multicolumn{4}{|c|}{ Performance (\% cost increase) heuristics } \\
\hline & & & & & & & A & B & $\mathrm{C}$ & $\mathrm{D}$ \\
\hline 1 & 9 & 207 & 207 & 12 & 12.43 & 12.43 & 20.6 & 18.1 & 0.3 & 11.4 \\
\hline 2 & 10 & 181 & 181 & 20 & 27.88 & 27.88 & 26.5 & 14.4 & 0.1 & 5.0 \\
\hline 3 & 12 & 264 & 264 & 22 & 20.17 & 20.17 & 22.4 & 16.0 & 0.0 & 5.3 \\
\hline 4 & 12 & 220 & 220 & 24 & 19.33 & 19.33 & 10.4 & 4.7 & 0.0 & 2.5 \\
\hline 5 & 9 & 183 & 183 & 25 & 22.68 & 22.68 & 16.7 & 10.2 & 0.4 & 9.4 \\
\hline 6 & 12 & 187 & 188 & 26 & 14.16 & 14.14 & 15.2 & 10.8 & 0.1 & 5.2 \\
\hline 7 & 10 & 168 & 168 & 26 & 18.95 & 18.95 & 21.3 & 13.4 & 0.3 & 6.4 \\
\hline 8 & 8 & 126 & 126 & 29 & 45.90 & 45.90 & 11.2 & 1.4 & 0.7 & 1.3 \\
\hline 9 & 16 & 155 & 155 & 30 & 21.75 & 21.75 & 23.1 & 2.8 & 0.1 & 2.7 \\
\hline 10 & 10 & 129 & 129 & 33 & 35.46 & 35.46 & 12.1 & 2.3 & 0.1 & 1.7 \\
\hline 11 & 13 & 156 & 156 & 33 & 20.50 & 20.50 & 26.4 & 12.7 & 1.9 & 9.1 \\
\hline 12 & 9 & 87 & 87 & 34 & 44.89 & 44.89 & 14.3 & 0.7 & 0.5 & 0.6 \\
\hline 13 & 11 & 256 & 256 & 34 & 23.27 & 23.27 & 6.2 & 1.4 & 0.4 & 1.2 \\
\hline 14 & 16 & 266 & 266 & 34 & 15.61 & 15.61 & 13.2 & 10.0 & 0.1 & 9.5 \\
\hline 15 & 12 & 267 & 267 & 35 & 14.49 & 14.49 & 16.4 & 4.7 & 0.1 & 3.4 \\
\hline 16 & 14 & 132 & 132 & 37 & 31.01 & 31.01 & 3.7 & 0.8 & 0.3 & 0.6 \\
\hline 17 & 14 & 220 & 220 & 38 & 11.40 & 11.40 & 28.7 & 16.8 & 3.3 & 15.2 \\
\hline 18 & 12 & 149 & 149 & 38 & 25.83 & 25.83 & 18.1 & 3.4 & 0.4 & 3.3 \\
\hline 19 & 12 & 141 & 141 & 40 & 23.60 & 23.60 & 11.7 & 11.0 & 0.6 & 0.8 \\
\hline 20 & 11 & 144 & 144 & 40 & 21.77 & 21.77 & 19.8 & 5.0 & 2.8 & 5.0 \\
\hline 21 & 13 & 147 & 147 & 40 & 28.64 & 28.64 & 13.3 & 1.3 & 6.4 & 1.2 \\
\hline 22 & 14 & 151 & 151 & 41 & 35.96 & 35.96 & 21.6 & 2.5 & 0.1 & 2.0 \\
\hline 23 & 13 & 105 & 105 & 41 & 35.34 & 35.34 & 15.5 & 4.7 & 1.0 & 0.9 \\
\hline 24 & 9 & 110 & 110 & 42 & 30.54 & 30.54 & 22.0 & 7.2 & 0.2 & 6.5 \\
\hline 25 & 14 & 278 & 278 & 43 & 14.54 & 14.54 & 3.0 & 1.5 & 0.1 & 1.2 \\
\hline 26 & 14 & 168 & 168 & 43 & 31.29 & 31.29 & 16.5 & 1.4 & 0.0 & 0.8 \\
\hline 27 & 12 & 187 & 187 & 43 & 24.84 & 24.84 & 2.2 & 0.8 & 0.0 & 0.4 \\
\hline 28 & 15 & 177 & 177 & 46 & 23.76 & 23.76 & 2.3 & 1.6 & 0.1 & 0.4 \\
\hline 29 & 13 & 102 & 102 & 47 & 47.80 & 47.80 & 7.7 & 0.4 & 0.4 & 0.3 \\
\hline 30 & 16 & 182 & 182 & 47 & 24.98 & 24.98 & 12.8 & 9.3 & 0.2 & 3.0 \\
\hline
\end{tabular}


- Examples 31-60: capacity utilization is between $50 \%$ and $75 \%$;

- Examples 61-90: capacity utilization is between $75 \%$ and $100 \%$;

- Examples 90-120: cycle time $T_{\mathrm{IC}}$ is smaller than $T_{\min }$.

\subsection{Evaluation of the results}

To evaluate quality of the solution, we compare the cost of the heuristic solution with that of the solution $\mathrm{TC}^{*}$ determined by the exact algorithm developed by Tang and Teunter (2006).

$\Delta=\frac{\mathrm{TC}(\text { heuristic })-\mathrm{TC}^{*}}{\mathrm{TC}^{*}}$.

The exact algorithm was written on $\mathrm{C}$ programming language using the callable library of CPLEX 8.0. The code was edited and compiled using Microsoft Visual Studio.NET 2003, while the experiments were designed and performed on a DELL desktop with an Intel Pentium4 processor at $3.0 \mathrm{GHz}$ and 512 RAM. We remark that although CPLEX currently incorporates the state-of-theart code for MIP, deriving the optimal plan was very time consuming for some examples with high capacity utilization, and therefore the algorithm needed to be terminated before an optimal solution was reached. We decided to terminate the branching of the MIP (for a given cycle time) after $15 \mathrm{~min}$. This was based on observing no significant further improvement after the first few minutes of branching for any of the instances.

\subsection{Observations}

The results for all 120 examples are given in Tables 2-5. Note that in each table, the examples are ranked in ascending order of capacity. An “*” before the example number identifies that the exact algorithm had to be terminated before finding the optimal solution for that example, and hence that $\mathrm{TC}^{*}$ is not the (guaranteed) minimum cost but an upper bound for it. With increasing capacity utilization (CU), it obviously becomes more difficult to derive the exact optimal solution.

The results are also summarized in Table 6, where the average performances of the algorithms for the four different $\mathrm{CU}$ groups are reported. Note that the average best performances among Heuristics $B$ and $C$ and among $B, C$ and $D$ are also reported there, as it appears from Tables 2-5 that at least one of these two heuristics performs well in almost all examples. This will be discussed further below.

The first observation is that the performance of Heuristic A, which stops with the initial solution, is poor. For all CU groups, the average cost increase is more than $10 \%$ and the overall cost increase is $12.01 \%$. Hence, an improvement step is essential.

The preferred type of improvement, swapping production sequencing (Heuristic B) or inserting slack time

Table 3

Output for examples 31-60 with capacity utilization $50<\mathrm{CU}<75 \%$

\begin{tabular}{|c|c|c|c|c|c|c|c|c|c|c|}
\hline \multirow[t]{2}{*}{$i$} & \multirow[t]{2}{*}{$T_{\min }$} & \multirow[t]{2}{*}{$T_{\mathrm{IC}}$} & \multirow[t]{2}{*}{$T^{*}$} & \multirow[t]{2}{*}{$\mathrm{CU}(\%)$} & \multirow[t]{2}{*}{ IC } & \multirow[t]{2}{*}{$\mathrm{TC}^{*}$} & \multicolumn{4}{|c|}{ Performance (\% cost increase) heuristics } \\
\hline & & & & & & & A & B & $\mathrm{C}$ & $\mathrm{D}$ \\
\hline 31 & 17 & 337 & 337 & 50 & 11.45 & 11.45 & 1.4 & 0.8 & 0.2 & 0.6 \\
\hline 32 & 18 & 122 & 122 & 50 & 31.14 & 31.14 & 18.5 & 4.8 & 0.6 & 2.4 \\
\hline 33 & 15 & 165 & 165 & 51 & 27.14 & 27.14 & 18.9 & 8.3 & 2.1 & 7.9 \\
\hline 34 & 13 & 152 & 152 & 53 & 19.35 & 19.35 & 7.2 & 5.9 & 0.2 & 5.7 \\
\hline 35 & 19 & 201 & 201 & 53 & 18.87 & 18.87 & 4.7 & 3.0 & 0.1 & 0.1 \\
\hline 36 & 17 & 158 & 158 & 54 & 20.91 & 20.91 & 11.2 & 6.9 & 1.7 & 3.9 \\
\hline 37 & 12 & 142 & 142 & 55 & 27.42 & 27.42 & 13.9 & 1.5 & 1.2 & 1.4 \\
\hline 38 & 19 & 185 & 185 & 56 & 15.24 & 15.24 & 2.6 & 1.3 & 0.8 & 0.4 \\
\hline 39 & 19 & 173 & 173 & 56 & 23.64 & 23.64 & 7.8 & 6.3 & 0.2 & 5.4 \\
\hline 40 & 17 & 155 & 155 & 57 & 27.87 & 27.87 & 22.9 & 8.4 & 3.4 & 3.7 \\
\hline 41 & 16 & 128 & 127 & 57 & 40.54 & 41.29 & 14.8 & 0.7 & 2.4 & 0.2 \\
\hline 42 & 14 & 327 & 327 & 58 & 11.10 & 11.10 & 3.5 & 2.3 & 0.1 & 1.9 \\
\hline 43 & 21 & 137 & 137 & 59 & 43.69 & 43.69 & 4.0 & 0.7 & 0.0 & 0.6 \\
\hline 44 & 20 & 184 & 184 & 60 & 24.55 & 24.55 & 3.4 & 1.2 & 1.1 & 1.1 \\
\hline 45 & 16 & 106 & 106 & 61 & 30.88 & 30.88 & 36.6 & 2.6 & 1.3 & 1.8 \\
\hline 46 & 21 & 107 & 107 & 61 & 30.63 & 30.63 & 7.7 & 6.1 & 0.1 & 1.4 \\
\hline 47 & 24 & 111 & 111 & 61 & 31.29 & 31.29 & 10.8 & 2.4 & 2.2 & 0.6 \\
\hline 48 & 20 & 105 & 105 & 61 & 28.01 & 28.01 & 7.1 & 5.3 & 0.2 & 1.1 \\
\hline 49 & 21 & 142 & 142 & 62 & 35.97 & 35.97 & 7.1 & 1.8 & 1.8 & 1.8 \\
\hline 50 & 18 & 98 & 98 & 63 & 31.79 & 31.79 & 15.1 & 1.8 & 0.2 & 0.3 \\
\hline 51 & 17 & 104 & 104 & 64 & 59.53 & 59.53 & 1.2 & 0.2 & 0.2 & 0.2 \\
\hline 52 & 19 & 219 & 219 & 64 & 17.71 & 17.71 & 4.2 & 1.8 & 2.1 & 1.3 \\
\hline 53 & 26 & 135 & 135 & 66 & 25.83 & 25.83 & 10.2 & 0.3 & 0.4 & 0.2 \\
\hline 54 & 33 & 150 & 150 & 68 & 25.81 & 25.81 & 17.0 & 0.4 & 2.9 & 0.4 \\
\hline 55 & 29 & 226 & 226 & 70 & 19.84 & 19.84 & 9.2 & 3.6 & 5.8 & 1.4 \\
\hline 56 & 24 & 180 & 180 & 73 & 27.57 & 27.57 & 10.3 & 1.1 & 10.3 & 1.1 \\
\hline 57 & 35 & 177 & 178 & 74 & 23.08 & 23.13 & 23.2 & 4.2 & 9.3 & 1.6 \\
\hline 58 & 28 & 184 & 184 & 74 & 25.65 & 25.65 & 3.1 & 1.0 & 1.3 & 0.9 \\
\hline 59 & 27 & 148 & 148 & 75 & 27.51 & 27.51 & 10.9 & 6.3 & 4.2 & 1.9 \\
\hline 60 & 35 & 213 & 213 & 75 & 22.43 & 22.43 & 17.1 & 0.6 & 12.6 & 0.5 \\
\hline
\end{tabular}


Table 4

Output for examples 61-90 with capacity utilization $75 \%<\mathrm{CU}<100 \%$

\begin{tabular}{|c|c|c|c|c|c|c|c|c|c|c|}
\hline \multirow[t]{2}{*}{$i$} & \multirow[t]{2}{*}{$T_{\min }$} & \multirow[t]{2}{*}{$T_{I C}$} & \multirow[t]{2}{*}{$T^{*}$} & \multirow[t]{2}{*}{$\mathrm{CU}(\%)$} & \multirow[t]{2}{*}{ IC } & \multirow[t]{2}{*}{$\mathrm{TC}^{*}$} & \multicolumn{4}{|c|}{ Performance (\% cost increase) heuristics } \\
\hline & & & & & & & A & B & $\mathrm{C}$ & $\mathrm{D}$ \\
\hline 61 & 36 & 169 & 169 & 75 & 28.32 & 28.32 & 2.5 & 0.7 & 0.4 & 0.6 \\
\hline 62 & 37 & 231 & 231 & 76 & 14.68 & 14.68 & 7.0 & 2.7 & 6.2 & 2.7 \\
\hline 63 & 37 & 167 & 167 & 76 & 31.04 & 31.04 & 16.9 & 2.8 & 1.9 & 2.8 \\
\hline 64 & 32 & 99 & 99 & 77 & 42.33 & 42.33 & 3.6 & 1.9 & 3.6 & 1.9 \\
\hline *65 & 37 & 189 & 189 & 77 & 20.46 & 20.50 & 8.9 & 5.8 & 8.9 & 5.8 \\
\hline 66 & 33 & 145 & 145 & 79 & 33.84 & 33.84 & 12.5 & 3.3 & 12.0 & 0.3 \\
\hline 67 & 36 & 125 & 126 & 81 & 29.69 & 29.69 & 16.5 & 0.5 & 4.4 & 0.5 \\
\hline 68 & 48 & 126 & 126 & 81 & 31.78 & 31.78 & 25.7 & 0.6 & 12.0 & 0.6 \\
\hline 69 & 48 & 108 & 108 & 82 & 21.86 & 21.86 & 16.0 & 2.3 & 8.1 & 1.8 \\
\hline *70 & 38 & 168 & 167 & 82 & 22.54 & 22.73 & 20.2 & 12.0 & 7.8 & 9.4 \\
\hline *71 & 49 & 170 & 170 & 82 & 13.93 & 13.93 & 6.8 & 0.6 & 6.8 & 0.4 \\
\hline 72 & 37 & 96 & 96 & 83 & 36.47 & 36.47 & 1.8 & 0.4 & 1.8 & 0.3 \\
\hline 73 & 44 & 93 & 93 & 83 & 45.39 & 45.39 & 3.2 & 0.5 & 1.2 & 0.5 \\
\hline *74 & 49 & 283 & 283 & 86 & 13.49 & 13.49 & 18.4 & 6.9 & 6.9 & 1.7 \\
\hline *75 & 64 & 158 & 158 & 88 & 30.62 & 30.62 & 6.5 & 1.8 & 1.5 & 1.8 \\
\hline *76 & 57 & 90 & 90 & 89 & 39.00 & 39.00 & 2.4 & 0.7 & 1.9 & 0.7 \\
\hline *77 & 63 & 136 & 136 & 89 & 35.58 & 35.58 & 9.8 & 0.9 & 4.1 & 0.9 \\
\hline 78 & 77 & 246 & 246 & 89 & 23.29 & 23.29 & 16.6 & 5.8 & 15.2 & 5.6 \\
\hline *79 & 78 & 258 & 259 & 90 & 15.64 & 15.85 & 11.1 & 4.0 & 11.1 & 4.0 \\
\hline *80 & 100 & 132 & 131 & 92 & 24.62 & 24.63 & 4.9 & 0.7 & 4.9 & 0.7 \\
\hline *81 & 108 & 141 & 141 & 92 & 25.96 & 25.96 & 12.2 & 0.2 & 10.8 & 0.2 \\
\hline *82 & 97 & 179 & 180 & 92 & 20.19 & 20.27 & 11.7 & 1.8 & 11.7 & 1.5 \\
\hline *83 & 141 & 146 & 146 & 93 & 22.79 & 22.85 & 2.6 & 0.2 & 2.6 & 0.2 \\
\hline *84 & 153 & 168 & 169 & 95 & 21.59 & 21.59 & 9.9 & 1.2 & 9.9 & 1.2 \\
\hline *85 & 189 & 224 & 225 & 95 & 24.10 & 24.11 & 2.7 & 0.8 & 2.7 & 0.8 \\
\hline *86 & 232 & 134 & 232 & 96 & 22.56 & 31.39 & 16.2 & 0.1 & 16.2 & 0.1 \\
\hline *87 & 175 & 145 & 175 & 97 & 24.61 & 25.11 & 5.6 & 0.6 & 5.6 & 0.6 \\
\hline *88 & 424 & 165 & 425 & 98 & 19.71 & 38.15 & 10.8 & -0.2 & 10.8 & -0.2 \\
\hline *89 & 483 & 162 & 484 & 98 & 23.36 & 46.34 & 14.9 & 8.1 & 14.9 & 8.1 \\
\hline *90 & 548 & 154 & 549 & 99 & 27.08 & 61.18 & 29.5 & 17.3 & 29.5 & 17.3 \\
\hline
\end{tabular}

(Heuristic C), depends on the capacity utilization. Table 6 shows that the preference shifts from Heuristic $C$ to be with increasing CU. This is logical, and simply shows that Heuristic C needs 'enough' capacity to insert slack time in order to perform well. The detailed results in Tables 2-5 show that, roughly, Heuristic $C$ performs better with CU below $65 \%$ and Heuristic B performs better above 65\%.

As mentioned above, Tables 2-5 further show that either Heuristic B or C performs well for almost all cases. More precisely, there are just 7 (out of 120) examples where the cost increase for the better of the two solutions is more than 5\%. The average cost increase over all examples is only $1.53 \%$, as reported in Table 6 .

Heuristic D, which applies both improvement steps sequentially, performs better than the best of Heuristics B and $\mathrm{C}$ for some examples, but only marginally. The average cost increase over all examples is only $1.36 \%$.

\section{Discussion and conclusions}

In this paper we have developed and investigated 4 heuristics for the ELSPR problem. These heuristics all find an initial solution and then do or do not apply two further improvement steps by swapping production sequences or inserting slack time. The numerical results show that applying no improvement steps results in poor performance. Which improvement step is better depends on the capacity utilization, with $65 \%$ roughly being the cut-off point below which inserting slack time (Heuristic C) is better, and above which swapping production sequences (Heuristic B) is better. Moreover, in most cases applying both these heuristics and selecting the best solution works better than applying the single complex heuristic that uses both steps sequentially (Heuristic D). The average cost increase of the best (of $B$ and $C$ ) solutions compared with that found by the exact algorithm from Tang and Teunter (2006) is $1.53 \%$. Another small reduction to $1.36 \%$ on average is obtained by taking the best solution of B-D.

The contribution of this research result is two-fold. First, due to their simplicity, the above-mentioned heuristics can be readily implemented in practice. With an increasing number of companies conducting remanufacturing business and encountering ELSPR problem, our heuristics provide a good tool for making sound decisions. Secondly, similar as in the conventional ELSP research, there is a great potential to develop new heuristics and scheduling policies in dealing with ELSPR problem, such as base-period policy, power-of-two policy. In this case, our numerical examples (available from the authors) and the associated lower bound solutions can be used as a benchmark for the research.

Besides considering different policies in dealing with the ELSPR problem, there is also a need to develop models for the case with separate production lines for 
Table 5

Output for examples 91-120 with $T_{\min }>T_{\mathrm{IC}}$

\begin{tabular}{|c|c|c|c|c|c|c|c|c|c|c|}
\hline \multirow[t]{2}{*}{$i$} & \multirow[t]{2}{*}{$T_{\min }$} & \multirow[t]{2}{*}{$T_{\mathrm{IC}}$} & \multirow[t]{2}{*}{$T^{*}$} & \multirow[t]{2}{*}{ CU $(\%)$} & \multirow[t]{2}{*}{ IC } & \multirow[t]{2}{*}{$\mathrm{TC}^{*}$} & \multicolumn{4}{|c|}{ Performance (\% cost increase) heuristics } \\
\hline & & & & & & & A & B & C & D \\
\hline$* 91$ & 45 & 37 & 45 & 87 & 42.52 & 43.42 & 1.2 & 0.1 & 1.2 & 0.1 \\
\hline *92 & 38 & 35 & 38 & 89 & 50.33 & 50.55 & 5.1 & 1.4 & 5.1 & 1.4 \\
\hline *93 & 38 & 33 & 38 & 90 & 61.07 & 61.68 & 0.6 & 0.3 & 0.6 & 0.3 \\
\hline *94 & 55 & 40 & 55 & 92 & 48.70 & 51.17 & 14.2 & 2.6 & 14.2 & 2.6 \\
\hline *95 & 62 & 45 & 62 & 92 & 57.61 & 66.24 & 14.7 & 0.0 & 14.7 & 0.0 \\
\hline *96 & 49 & 44 & 49 & 93 & 43.76 & 44.08 & 1.7 & 1.0 & 1.7 & 1.0 \\
\hline *97 & 66 & 46 & 66 & 94 & 43.18 & 46.12 & 24.5 & 1.5 & 24.5 & 1.5 \\
\hline *98 & 65 & 43 & 65 & 94 & 31.74 & 34.61 & 10.8 & 2.7 & 10.8 & 2.7 \\
\hline *99 & 58 & 38 & 58 & 94 & 41.05 & 44.69 & 16.0 & 4.0 & 16.0 & 4.0 \\
\hline *100 & 66 & 58 & 66 & 94 & 36.31 & 36.59 & 24.5 & 0.3 & 24.5 & 0.3 \\
\hline *101 & 75 & 37 & 75 & 95 & 40.86 & 51.22 & 8.4 & 0.1 & 8.4 & 0.1 \\
\hline *102 & 74 & 48 & 74 & 95 & 55.26 & 60.73 & 30.6 & 1.2 & 30.6 & 1.2 \\
\hline *103 & 69 & 67 & 69 & 95 & 38.27 & 38.30 & 7.9 & 1.4 & 7.9 & 1.4 \\
\hline *104 & 105 & 89 & 105 & 96 & 24.82 & 25.18 & 18.8 & 2.6 & 18.8 & 2.6 \\
\hline *105 & 114 & 94 & 114 & 96 & 25.64 & 26.14 & 11.3 & 9.3 & 11.3 & 9.3 \\
\hline *106 & 128 & 29 & 128 & 96 & 58.54 & 137.44 & 3.6 & 0.3 & 3.6 & 0.3 \\
\hline *107 & 115 & 59 & 115 & 96 & 45.19 & 55.90 & 15.5 & 1.6 & 15.5 & 1.6 \\
\hline *108 & 113 & 47 & 113 & 97 & 34.63 & 48.58 & 11.8 & 4.6 & 11.8 & 4.6 \\
\hline *109 & 126 & 35 & 126 & 97 & 53.03 & 102.39 & 13.7 & 0.8 & 13.7 & 0.8 \\
\hline$* 110$ & 132 & 106 & 132 & 97 & 15.68 & 16.08 & 5.8 & 0.7 & 5.8 & 0.7 \\
\hline *111 & 111 & 38 & 111 & 97 & 38.75 & 62.81 & 3.3 & 1.2 & 3.3 & 1.2 \\
\hline *112 & 199 & 73 & 199 & 98 & 35.26 & 54.84 & 0.8 & 0.3 & 0.8 & 0.2 \\
\hline *113 & 187 & 43 & 187 & 98 & 43.24 & 100.07 & 0.3 & 0.0 & 0.3 & 0.0 \\
\hline *114 & 204 & 88 & 204 & 98 & 26.82 & 36.88 & 10.4 & 1.2 & 10.4 & 1.2 \\
\hline *115 & 320 & 73 & 320 & 99 & 35.34 & 81.72 & 6.7 & 0.4 & 6.7 & 0.4 \\
\hline *116 & 377 & 75 & 377 & 99 & 32.04 & 83.79 & 14.5 & 5.5 & 14.5 & 5.5 \\
\hline *117 & 410 & 84 & 410 & 99 & 29.42 & 74.84 & 2.7 & 1.9 & 2.7 & 1.9 \\
\hline *118 & 408 & 58 & 408 & 99 & 41.25 & 148.39 & 1.7 & 0.8 & 1.7 & 0.8 \\
\hline *119 & 736 & 50 & 736 & 99 & 46.13 & 342.47 & 18.9 & 1.4 & 18.9 & 1.4 \\
\hline *120 & 498 & 73 & 498 & 99 & 32.98 & 115.39 & 32.6 & 3.6 & 32.6 & 3.6 \\
\hline
\end{tabular}

Table 6

Summary of the average performance (\% cost increase) results

\begin{tabular}{lllllll}
\hline & A & B & C & D & $\operatorname{Min}(B, C)$ & $\operatorname{Min}(B-D)$ \\
\hline Examples 1-30 & 15.17 & 6.38 & 0.70 & 3.88 & 0.53 & 0.52 \\
Examples 31-60 & 10.85 & 3.07 & 2.31 & 1.73 & 1.20 & 0.84 \\
Examples 61-90 & 10.92 & 2.84 & 7.85 & 2.43 & 2.65 & 2.33 \\
Examples 91-120 & 11.09 & 1.76 & 11.08 & 1.75 & 1.76 & 1.76 \\
& & & & & & \\
Overall & 12.01 & 3.51 & 5.48 & 2.45 & 1.53 & 1.36 \\
\hline
\end{tabular}

manufacturing and remanufacturing, in contrast to the case with a single line considered here. Separate lines do no imply that the scheduling problems separate as well, since items produced on both lines enter a common serviceable stock. Development of both exact and heuristic approaches is of interest.

\section{References}

Bomberger, E.E., 1966. A dynamic programming approach to a lot size scheduling problem. Management Sciences 12 (11), 778-784.
Davis, S.G., 1990. Scheduling economic lot size production runs Management Science 36 (8), 985-998.

Elmaghraby, S.E., 1978. The economic lot scheduling problem (ELSP): Review and extensions. Management Science 24 (6), 587-598.

Guide, V.D.R., 2000. Production planning and control for remanufacturing: Industry practice and research needs. Journal of Operations Management 18 (4), 467-483.

Hsu, W.L., 1983. On the general feasibility of scheduling lot sizes of several products on one machine. Management Sciences 29, 93-105.

Koh, S.-G., Hwang, H., Sohn, K.-I., Ko, C.-S., 2002. An optimal ordering and recovery policy for reusable items. Computers and Industrial Engineering 43, 59-73.

Richter, K., 1996. The EOQ and waste disposal model with variable setup numbers. European Journal of Operational Research 95, 313-324.

Tang, O., Teunter, R.H., 2006. Economic lot scheduling problem with returns. Production and Operations Management 15 (4), 488-497.

Teunter, R.H., 2001. Economic ordering quantities for recoverable item inventory systems. Naval Research Logistics 48, 484-495.

Teunter, R.H., 2004. Lot-sizing for inventory systems with product recovery. Computers and Industrial Engineering 46 (3), 431-441.

Teunter, R.H., Van der Laan, E.A., Inderfurth, K., 2000. On how to set the holding cost rates in inventory models with returned items that can be remanufactured. Omega 28 (4), 409-415. 